Patterned structures of in situ size controlled CdS nanocrystals in a polymer matrix under UV irradiation

This content has been downloaded from IOPscience. Please scroll down to see the full text. 2009 Nanotechnology 20155302

(http://iopscience.iop.org/0957-4484/20/15/155302)

View the table of contents for this issue, or go to the journal homepage for more

Download details:

IP Address: 141.117.125.76

This content was downloaded on 16/06/2015 at 03:35

Please note that terms and conditions apply. 


\title{
Patterned structures of in situ size controlled CdS nanocrystals in a polymer matrix under UV irradiation
}

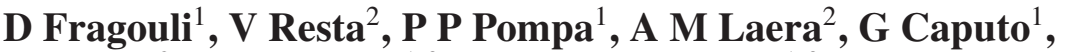 \\ L Tapfer ${ }^{2}, \mathbf{R}$ Cingolani $^{1,3}$ and A Athanassiou ${ }^{1,3}$ \\ ${ }^{1}$ NNL-National Nanotechnology Laboratory, INFM, CNR, Via Arnesano, 73100 Lecce, \\ Italy \\ 2 ENEA, Centro Ricerche Brindisi, SS7 Appia Km 706, I-72100 Brindisi, Italy \\ ${ }^{3}$ IIT-Italian Institute of Technology, Via Morego 30, Genova, Italy \\ E-mail: despina.fragouli@unile.it
}

Received 22 December 2008, in final form 11 February 2009

Published 24 March 2009

Online at stacks.iop.org/Nano/20/155302

\begin{abstract}
A method of in situ formation of patterns of size controlled CdS nanocrystals in a polymer matrix by pulsed UV irradiation is presented. The films consist of Cd thiolate precursors with different carbon chain lengths embedded in TOPAS polymer matrices. Under UV irradiation the precursors are photolyzed, driving to the formation of CdS nanocrystals in the quantum size regime, with size and concentration defined by the number of incident UV pulses, while the host polymer remains macroscopically/microscopically unaffected. The emission of the formed nanocomposite materials strongly depends on the dimensions of the CdS nanocrystals, thus, their growth at the different phases of the irradiation is monitored using spatially resolved photoluminescence by means of a confocal microscope. X-ray diffraction measurements verified the existence of the CdS nanocrystals, and defined their crystal structure for all the studied cases. The results are reinforced by transmission electron microscopy. It is proved that the selection of the precursor determines the efficiency of the procedure, and the quality of the formed nanocrystals. Moreover it is demonstrated that there is the possibility of laser induced formation of well-defined patterns of CdS nanocrystals, opening up new perspectives in the development of nanodevices.
\end{abstract}

(Some figures in this article are in colour only in the electronic version)

\section{Introduction}

In recent years, the study of novel materials based on semiconductor nanocrystals (NCs) has attracted great fundamental and industrial interest, with many potential applications both in mesoscopic research and in the development of nanodevices. This is mainly due to their unique size dependent physical, chemical, and optical properties, known as the quantum confinement effect. In particular, the II-VI semiconductor NCs, have received much attention for potential applications in semiconductor lasers [1], optoelectronic nanodevices [2-8], and biological labeling $[9,10]$, due to tunable electronic band gaps depending on their size and shape $[11,12]$. In order to use these NCs in real applications it is necessary to stabilize them by means of inert inorganic or organic materials as matrices [13-17]. The incorporation/encapsulation of NCs in polymeric matrices is a very interesting approach, since the polymer host materials can be designed to yield a variety of bulk physical properties, while they exhibit long term stability, possess flexible processability [18], and prevent NC aggregation and degradation $[19,20]$. Moreover, the obtained nanocomposite materials combine special properties of both the polymeric and inorganic components, such as the mechanical and thermal properties of the former, and the tuneable optical and electronic features of the latter [18, 20].

Our approach focuses on the in situ localized formation of CdS NCs in the quantum size regime, by the use of pulsed UV laser irradiation of a polymer-precursor film. The size 
of the NCs is controlled by the number and the energy of the incident laser pulses, while the polymer matrix remains macroscopically and microscopically unaffected. The previous studies on this issue revealed the importance of the appropriate selection of the irradiation wavelength $[21,22]$ so as to form nanocomposite materials with well controlled size tuning of the CdS NCs, and narrow emission spectra, localized in welldefined areas of the film. In particular, it was proved that the use of a pulsed laser at $266 \mathrm{~nm}$ for the irradiation of the metal bis-dodecanthiolate precursor embedded in a TOPAS polymer matrix leads to the formation of high quality CdS NCs, by the mechanism of direct breakage of the $\mathrm{C}-\mathrm{S}$ bonds [22].

Here is presented a comparison between two metal thiolate precursors with different chain lengths, both giving CdS NCs embedded in a TOPAS polymer matrix, under pulsed laser irradiation at $266 \mathrm{~nm}$. For both cases, the formation of well dispersed CdS NCs occurs at the initial pulses. Moreover the NC size increases with an increasing number of incident pulses. This procedure is followed by the emission study of the irradiated areas, since CdS NCs smaller than $\sim 7-9 \mathrm{~nm}$ exhibit a blue shift to their emission as their size decreases, while for higher dimensions they show bulk behavior with an emission peak slightly above $500 \mathrm{~nm}$ (bulk band gap energy, $E_{\mathrm{g}}=2.4-2.45 \mathrm{eV}$ ) [23-25]. The difference between the two precursors concerns the speed of the nucleation and the surface defects of the formed NCs, explained by their differences in absorption and structure. By this study the pulse to pulse NC formation is optimized, giving accurate control of their dimensions in a localized area of the polymer matrix. Thus, the accuracy of the method is proved and improved. Following the next step for the use of the method for potential applications, the fabrication of precise CdS NC patterns into the polymer is demonstrated. The patterns become apparent only by observing the fluorescence images, while the polymer matrix remains macroscopically/microscopically unaffected. This study proves the potential of the method for spatially controlled fabrication and nanopatterning of NCs in polymer matrices with controlled size.

\section{Experimental details}

\subsection{Film preparation}

The metal precursors used are the cadmium bis-dodecanthiolate $\mathrm{Cd}\left(\mathrm{SC}_{12} \mathrm{H}_{25}\right)_{2},(\mathrm{C} 12)$, and cadmium bis-pentanthiolate $\mathrm{Cd}\left(\mathrm{SC}_{5}\right.$ $\left.\mathrm{H}_{11}\right)_{2}$, (C5), while the polymer is TOPAS ${ }^{\circledR}$, a thermoplastic cyclo-olefin copolymer consisting of ethylene and norbonene units, transparent in the visible. The chemical reaction formulae for the preparation of the precursors are described in detail in [26]. $20 \%$ by weight of the metal thiolate precursors was mixed with $80 \%$ by weight of TOPAS, and then diluted in toluene. The solutions were sonicated for $30 \mathrm{~min}$, in order to obtain the maximum dispersion of the insoluble C12, and C5. Next, they were cast in Petri capsules, and after the evaporation of the toluene, polymer-precursor films of thickness $\sim 200 \mu \mathrm{m}$ were formed.

\subsection{Irradiation of the films}

For the formation of the NCs in the polymer matrix, the films were irradiated with an increasing number of pulses of Nd:YAG laser (Quanta-Ray PRO-290-30, Spectra Physics) operating at the fourth harmonic, (wavelength $266 \mathrm{~nm}$, pulse duration $8 \mathrm{~ns}$, fluence $25 \mathrm{~mJ} \mathrm{~cm}^{-2}$, and repetition rate $2 \mathrm{~Hz}$ ). The laser beam was focused perpendicularly onto the surface of the samples to an area of $0.2 \mathrm{~cm}^{2}$. The series of irradiations were performed under air atmosphere and the laser fluence and repetition rate were low enough to prevent any structural changes on the surface of the polymer, but efficient for the formation of NCs.

\subsection{Characterization}

UV-visible absorption spectra were obtained using a UV-visNIR spectrophotometer (Cary 5000-Varian). Spatially resolved photoluminescence measurements have been performed on the irradiated films by a confocal microscope (Leica TCSSP5). The excitation wavelength $\left(\lambda_{\text {exc }}\right)$ was $405 \mathrm{~nm}$, and the irradiated areas were observed through a $20 \times, 0.70 \mathrm{NA}$ objective. For spectral analysis, the $\lambda_{\text {exc }}$ was $405 \mathrm{~nm}$, and the emission bandwidth was $10 \mathrm{~nm}$.

Low-magnification transmission electron microscopy (TEM) images of the laser-synthesized NCs were recorded with a JEOL JEM 1011 microscope operating at an accelerating voltage of $100 \mathrm{kV}$. The samples for TEM analysis were prepared by dropping a dilute solution of the irradiated part of the sample, dissolved in chloroform, onto carbon-coated copper grids and then allowing the solvent to evaporate. Next, the grids were immediately transferred to the TEM microscope and analyzed.

A statistical analysis of the size of the formed NCs was carried out using several widefield, low-magnification TEM images, with the help of Image-J software. For each sample, at least 150 particles were measured.

Low-resolution scanning electron microscopy (SEM) characterization of the surface of the C5-TOPAS and C12TOPAS films before and after UV irradiation was performed with a RAITH 150 EBL instrument. Typically, the images were acquired at low accelerating voltages (less than $5 \mathrm{kV}$ ) using short exposure times in order to avoid the charging of the samples.

For the crystal structure analysis of all the samples, $\mathrm{x}$ ray diffraction (XRD) measurements were performed using a $3 \mathrm{~kW}$ Bragg-Brentano diffractometer. The beam spot size had a diameter of about $8 \mathrm{~mm}$. The standard sample holders were made of aluminum. The films were mounted on (100) silicon wafers. All the measurements were made in the $\theta / 2 \theta$ configuration, with the same acquisition parameters $(25 \mathrm{~s}$ integration time, $0.05^{\circ}$ step size).

\section{Results and discussion}

\subsection{Irradiation of the films and study of the characteristics of the formed $\mathrm{NCs}$}

The polymer films containing cadmium bis-dodecanthiolate (C12) and cadmium bis-pentanthiolate (C5) were irradiated 


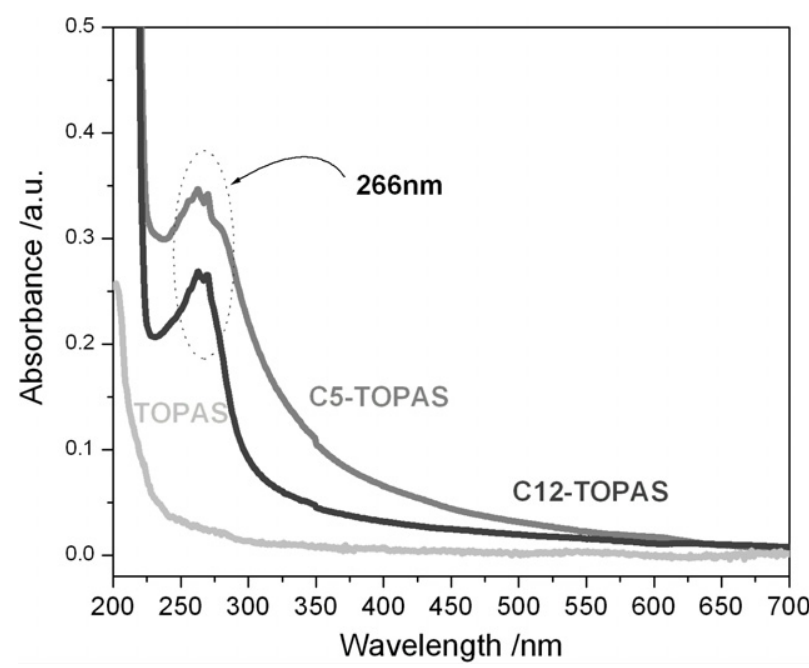

Figure 1. Normalized absorption spectra of the precursor-polymer, and of the bare polymer solutions.

with a pulsed UV laser at a wavelength of $266 \mathrm{~nm}$, and $2 \mathrm{~Hz}$ repetition rate. As shown in figure 1, the polymerprecursor samples exhibit maximum absorption at the selected wavelength, whereas the pure polymer samples absorb with very low efficiency. The laser fluence used for the irradiation is a critical parameter for the experimental procedure. It has to be high enough to initiate the NC formation but low enough to prevent any structural changes of the host polymer. Moreover, it is proved that above a certain fluence value, the formed CdS NCs immediately exhibit bulk properties. In fact, the study was conducted for films irradiated at 25 and $80 \mathrm{~mJ} \mathrm{~cm}^{-2}$. The emission spectra indicated that CdS NCs with bulk properties were formed at the very first pulse in the case of the high fluence for both kinds of precursor-polymer films, excluding the possibility of studying CdS NCs in the quantum size regime. However, the irradiation of the films with the lower fluence of $25 \mathrm{~mJ} \mathrm{~cm}{ }^{-2}$ allowed CdS NC formation with sizes that increased with an increasing number of pulses. Moreover, the SEM study of the samples, before and after irradiation at the specific fluence, verified the absence of any induced microscopic structural changes on the surface of the polymer. Indeed, figure 2 demonstrates representative images of the examined films, before and after UV irradiation. Hence, in order to study $\mathrm{NC}$ formation in the quantum regime the laser fluence was set at the value $25 \mathrm{~mJ} \mathrm{~cm}^{-2}$.

For the verification of the presence of CdS NCs after irradiation, and for their crystal structure analysis, XRD measurements were performed. The resulting patterns for C12TOPAS samples before and after irradiation with an increasing number of pulses from 6 to 80 are reported in figure 3(a). The study of the films confirmed the phase changes of the precursors to CdS NCs. With an increasing number of laser pulses, Bragg diffraction peaks appear that are related to the crystal structure of CdS, indicating the formation of both the hexagonal and the cubic phase. In particular, two diffraction peaks are associated to hexagonal CdS (JCPDS, PDF No. 800006), and another two to the cubic CdS (JCPDS, PDF No. 80-0019), with the (002) wurtzite and the (111) zincblende peaks overlapping. In figure 3(b) are reported theoretical simulations, done as described by Di Luccio et al [24] for the C12-TOPAS film irradiated with 20 laser pulses, and the corresponding experimental pattern. The calculation has been carried out by considering spherical CdS NCs with $14 \mathrm{~nm}$ diameter, estimated by using the Debye-Scherrer formula [27]. A relative good agreement between the experimental and theoretical data is obtained by assuming the coexistence of wurtzite and zincblende phases in the ratio of 55:45.

The evolution of the emission of the formed CdS NCs under a varying number of laser pulses is followed by spatially resolved photoluminescence measurements with a confocal microscope. Figure 4 shows the fluorescence images of the irradiated areas of two C5-TOPAS films, after 6 and 40 incident pulses, and in the inset are reported the fluorescence spectra from two selected areas of the films. After 6 pulses the density of the fluorescent NCs is very small, (figure 4(a)), and their emission peak close to $\lambda=440 \mathrm{~nm}$. After 40 laser pulses (figure 4(b)) the fluorescing area looks much denser, indicating increased $\mathrm{NC}$ formation, while the emission is shifted towards higher wavelengths with a peak at about $506 \mathrm{~nm}$ which coincides with the emission of the bulk CdS material.

For the accurate examination of the $\mathrm{CdS} \mathrm{NC}$ evolution, the spatially resolved emission study was carried out for all the irradiated films, and emission spectra from various areas of each fluorescence image were taken. Figures 5(a) and (b) show representative spectra for both precursors. It is clearly demonstrated that the emission of the samples changes dramatically after the first irradiation pulses, while an increase of the incident pulses causes a red shift to the emission, which is attributed to the increasing dimensions of the $\mathrm{CdS}$ NCs. Although this shift is apparent in the emission of both precursor films there is a clear difference in the width of their spectra. The C12-TOPAS films show statistically broader emission compared to the C5-TOPAS. The spectral deconvolution with three Gaussian functions (figures 6(a), (b)) of the emission spectra from the C12-TOPAS films after UV irradiation, revealed, apart from the peaks characteristic for the formation of the NCs, an additional broad emission band with a peak around $515 \mathrm{~nm}$ and an intensity that increases for an increasing number of incident pulses (figure 6(a)). This peak may be attributed to charge carrier recombination in trap states [24, 28-30]. In the case of the C5-TOPAS films the three Gaussian fit is not even possible for the emission spectra after 40 pulses, while in all the spectra the characteristic emission of CdS NCs is predominant. Thus, the trap state emission is very limited, or even nonexistent, in the C5-TOPAS films. Moreover, the spectral analysis revealed more than one peak that corresponds to $\mathrm{CdS} \mathrm{NCs}$ of different sizes, mostly in the case of C12-TOPAS films. This fact is attributed to the inhomogeneity of these films. Indeed, the C12 precursorTOPAS films look white, opalescent and less homogeneous than the C5 precursor-TOPAS films.

The detailed study of a large number of fluorescent areas of each film revealed the general trend of the $\mathrm{CdS}$ $\mathrm{NC}$ formation. Figure 7 demonstrates the dependence of the emission energy of the formed CdS NCs on the number of the 
incident UV pulses. For both precursor-TOPAS films, there is a significant emission shift of $69 \mathrm{~nm}$ (or $380 \mathrm{meV}$ ) upon increasing the number of pulses. In particular, the initial peak is centered at $2.83 \mathrm{eV}$ (corresponding to an emission wavelength of $437 \mathrm{~nm}$ ) while the final energy is close to $2.45 \mathrm{eV}(506 \mathrm{~nm})$, which corresponds to the bulk band gap energy of the CdS NCs. The main difference between the two precursors is that the CdS NC formation takes place in a fewer number of pulses in the case of the $\mathrm{C} 5$ precursor. In both cases, the band gap energy of the formed NCs decreases with an increasing number of incident laser pulses, a behavior characteristic of the quantum confinement effect, while after a specific number of pulses NCs with bulk properties start appearing. Above this threshold value NCs with bulk behavior, having a stable band gap energy close to $2.45 \mathrm{eV}$, and others that continue to alter their band gap energies upon an increasing number of incident pulses, can coexist on the same irradiated area. This phenomenon terminates when all the CdS NCs reach the size at which they exhibit bulk behavior. This happens after 80 pulses for the C12-TOPAS, and after 40 pulses for the C5-TOPAS films. The simultaneous existence of NCs with different sizes may be attributed to the inhomogeneity of the polymer-precursor films and of the laser beam. Thus, in order to form $\mathrm{CdS}$ NCs exclusively in the quantum confinement regime, the number of incident pulses should be less than 10 in the case of the C5-TOPAS, and less than 40 in the case of the C12-TOPAS films, for the specific experimental parameters.

After the emission studies, the irradiated nanocomposite films were examined with transmission electron microscopy (TEM). Figure 8(a) shows the TEM image of the C5-TOPAS film irradiated with $6 \mathrm{UV}$ pulses, where $\mathrm{CdS} \mathrm{NCs}$ with diameters around $4 \mathrm{~nm}$ are obvious in the magnified image. After 40 incident pulses the $\mathrm{NC}$ sizes have reached the bulk material (figure 8(b)) in perfect agreement with the emission measurements, while the same behavior is observed for the C12-TOPAS films, shifted to greater number of pulses. Figure 8(d) demonstrates the histogram of the CdS NC size distribution in both cases. In the films irradiated with 6 pulses it is clear that there exist small NCs with a maximum size distribution between 3 and $4 \mathrm{~nm}$, while after 40 pulses the formed NCs are mainly in the bulk region with a mean size of $10 \mathrm{~nm}$. The relatively broad distribution in both cases is attributed to the inhomogeneity of the spot of the laser beam and of the precursor's distribution in the polymer matrix.

The efficiency of the presented method is verified by comparing the sizes measured by the TEM images with the values extracted by the general theoretical model of the Brus equation [25]. This model describes the change of the band gap energy $\Delta E_{\mathrm{CdS}}$, related to the radius of the CdS NC $R_{\mathrm{CdS}}$ in the strong-confinement regime:

$$
\Delta E_{\mathrm{CdS}}=\left(\frac{\hbar^{2} \pi^{2}}{2 R_{\mathrm{CdS}}^{2}}\right)\left(\frac{1}{m_{\mathrm{e}}^{*}}+\frac{1}{m_{\mathrm{h}}^{*}}\right)-\frac{1.8 e^{2}}{\varepsilon R_{\mathrm{CdS}}}
$$

where $m_{\mathrm{e}}^{*}$ and $m_{\mathrm{h}}^{*}$ are the effective masses of the charge carrier (electron and hole, respectively); $\hbar$ the Planck's constant; and $\varepsilon$ the dielectric constant. By measuring the value of the $\Delta E_{\mathrm{CdS}}$ from the experimental data for both precursors, the CdS diameter is calculated. In agreement with the TEM study, (figure 8) after the initial 6 pulses the diameter is calculated to be about $4 \mathrm{~nm}$, and increases with an increasing number of laser pulses until it reaches the bulk material, with sizes close to $12 \mathrm{~nm}$.

\subsection{Mechanisms of the CdS formation}

This study reveals that both precursor molecules are efficient for the CdS NC formation under UV irradiation, with the main differences being the speed of this formation, and the broader emission in the case of the C12-TOPAS films. The mechanism followed for the CdS NC formation is considered to be photolysis since the irradiation wavelength used $(266 \mathrm{~nm})$ is at the peak of the C5/C12-TOPAS absorption. A fraction of the incident photons is absorbed by the precursors, and cause the $\mathrm{C}-\mathrm{S}$ chemical bond breakage as described by equation (2) [21].

$$
\begin{gathered}
\mathrm{Cd}(\mathrm{SR})_{2}+\mathrm{TOPAS} \stackrel{\mathrm{UV}}{\longrightarrow} \mathrm{CdS}+\mathrm{TOPAS}+\mathrm{SR}_{2} \\
\quad \text { (with } \mathrm{R}=\mathrm{C}_{12} \mathrm{H}_{25} \text { or } \mathrm{C}_{5} \mathrm{H}_{11} \text { ). }
\end{gathered}
$$

The C5-TOPAS samples exhibit a higher intensity of absorption compared to the C12-TOPAS, as already shown in the normalized spectra of figure 1 . Thus, under the same number of photons, a larger percentage is absorbed by the
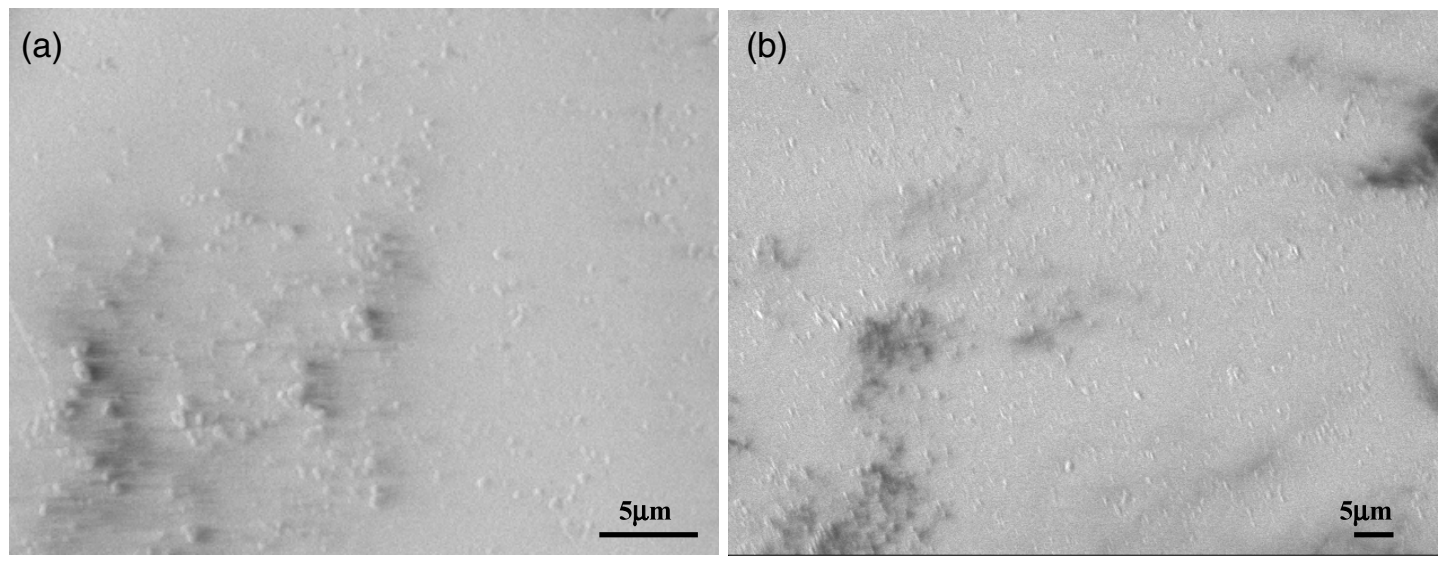

Figure 2. SEM images of the C12-TOPAS films, before irradiation (a) and after $80 \mathrm{UV}$ pulses (b). 

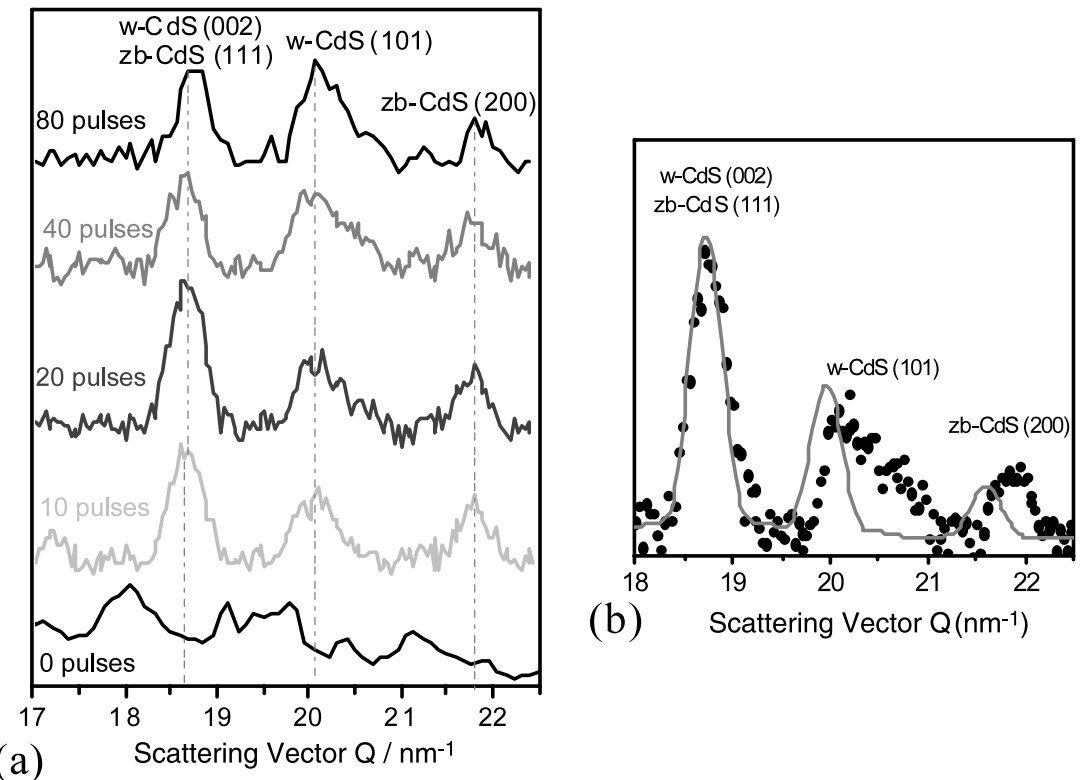

(a)

Figure 3. (a) XRD diffraction pattern of C12-TOPAS films. For every pattern is reported the corresponding number of irradiation pulses. (b) Simulated diffraction pattern of the C12-TOPAS film irradiated with 20 pulses (continuous line) obtained by assuming a mixed phase of $55 \% \mathrm{CdS}$ hexagonal and $45 \% \mathrm{CdS}$ cubic phase and by using the NC diameter value directly extracted by the experimental data (dotted line).

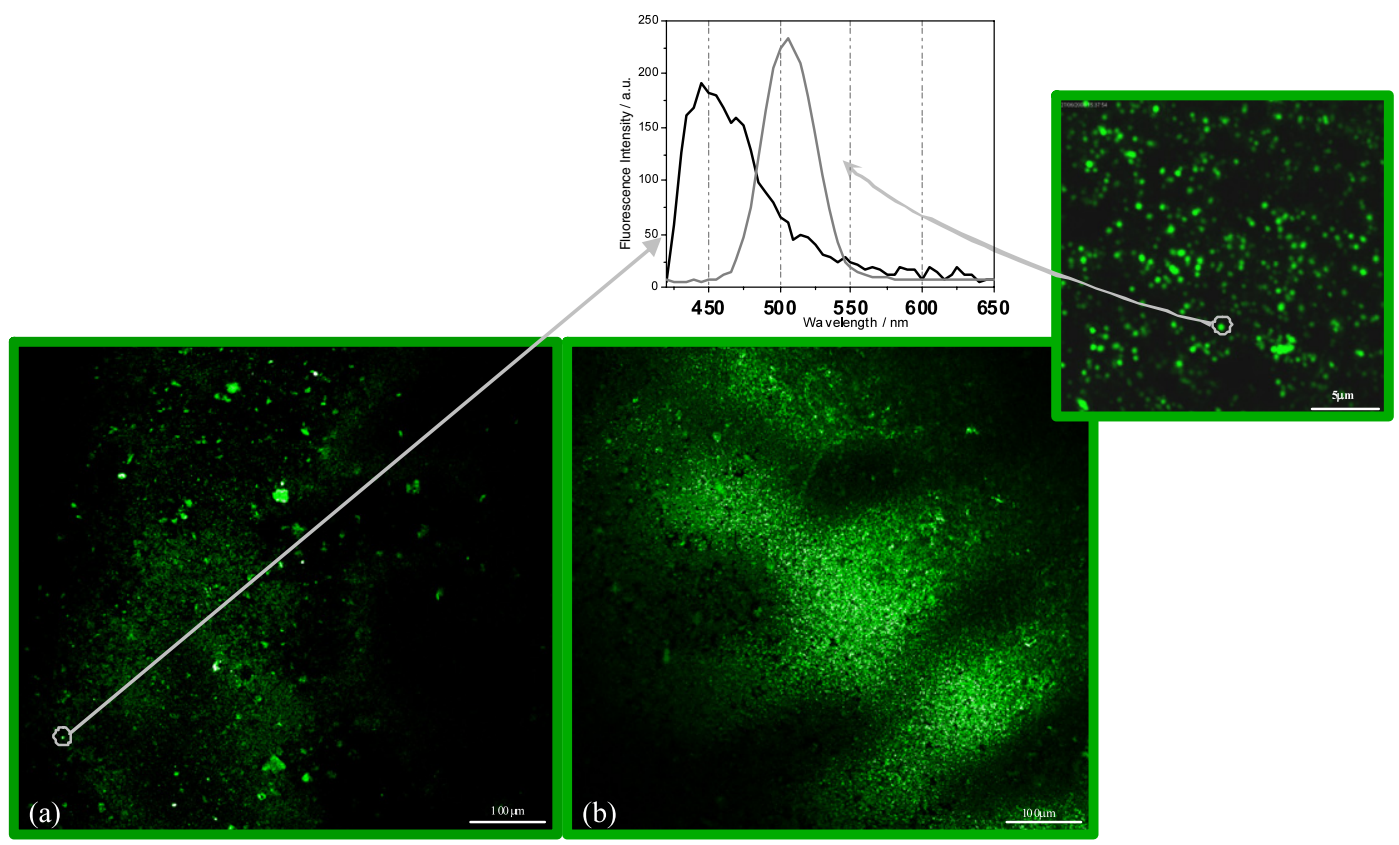

Figure 4. Photoluminescence images of CdS NCs formed after irradiation of the C5-TOPAS with 6 (a) and 40 (b) laser pulses at $266 \mathrm{~nm}$, fluence $25 \mathrm{~mJ} \mathrm{~cm}^{-2}$. Inset: Magnification of a specific area of case (b) and emission spectra of two bright areas selected from both images 'specified' as CdS NCs.

C5 precursor leading to a greater number of photolyzed $\mathrm{C}-\mathrm{S}$ bonds. Furthermore, the chain-chain interaction of the $\mathrm{C} 12$ $\mathrm{Cd}$ thiolates favors a lamellar structure arrangement of the $\mathrm{C} 12$ molecules, forming ordered domains. This is not observed in the smaller chain of the $\mathrm{C} 5$, where the molecules have different arrangement symmetry, mainly determined by the coordination chemistry of the Cd and S [24]. Conclusively, the arrangement of the $\mathrm{C} 12$ precursors is much more stable, thus more photons should be needed for their nucleation to CdS NCs. Since the same irradiation fluence has been used for all the samples, it is concluded that the photolysis and thus the NC nucleation is faster in the case of the precursor C5. Previous studies, carried out by thermal annealing of the same films [24], also revealed that in that case the NC formation is faster using $\mathrm{C} 5$ precursors.

Concerning the broader emission in the case of the C12-TOPAS samples, it is attributed to the charge carrier 

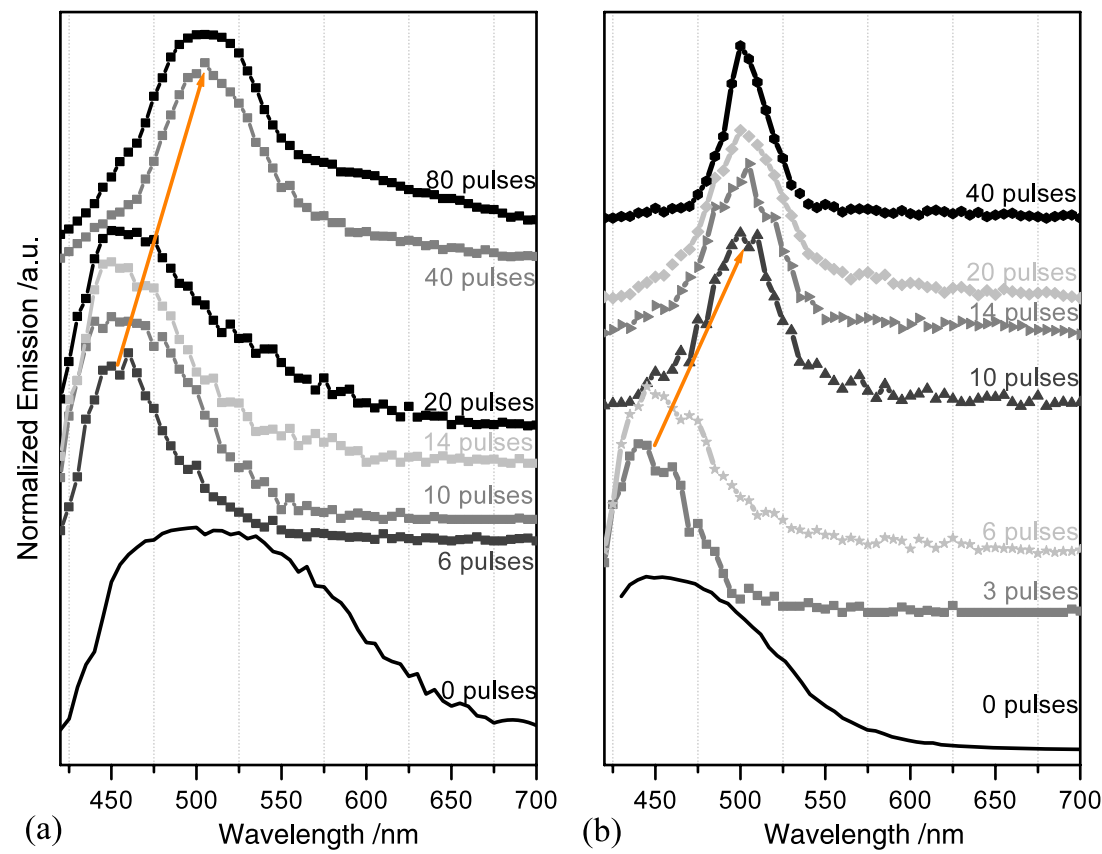

Figure 5. The change of the fluorescence spectra of C12-TOPAS (a) and C5-TOPAS (b) with an increasing number of incident laser pulses.
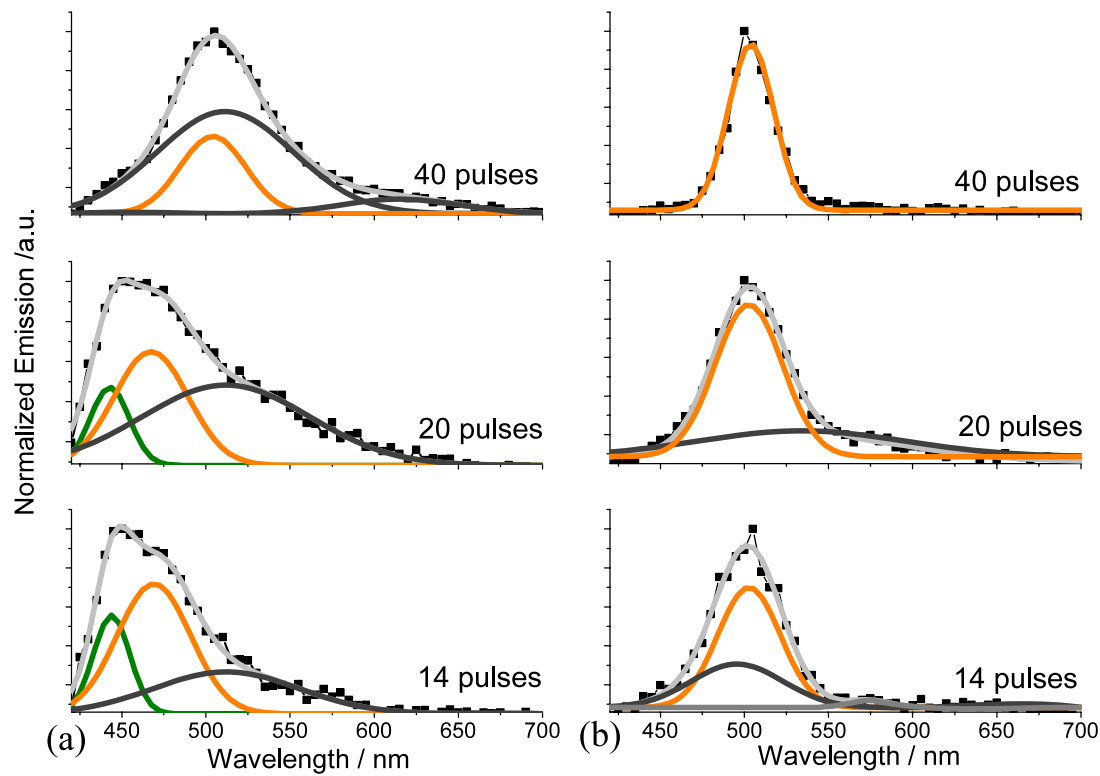

Figure 6. Spectral deconvolution of the emission of C12-TOPAS (a) and C5-TOPAS (b) films irradiated with 14, 20, and 40 pulses. The black symbols and the light gray curves correspond to the overall emission. The green and the orange curves correspond to the CdS NC emission, while the dark gray corresponds to the trap state emission.

recombination in trap states, as already assumed after the spectral deconvolution of the emission of the irradiated films. Generally, the trap state emission may be related in some cases to the low crystallinity [31,32], and in other cases to a sulfur excess or other defects at the interface of the NCs [24, 28-30]. In our case, surface defects due to sulfur excess and photoproducts of the polymer backbone chain are expected primarily on the surface of NCs obtained by UV irradiation of the C12-TOPAS films. This happens first because the byproducts of the $\mathrm{C} 12$ thiolate precursors
( $\mathrm{SR}_{2}$, with $\mathrm{R}=\mathrm{C}_{12} \mathrm{H}_{25}$ ), formed during the photolysis process, exhibit lower volatility, due to their higher molecular weight, compared to the C5 ones. Consequently, the evaporation/sublimation of those $\mathrm{C} 12$ products from the film during the CdS NC formation is less likely to happen [33]. Moreover, such thiolate byproducts of the $\mathrm{C} 12$ precursors are expected to have an energetically weaker $\mathrm{C}-\mathrm{S}$ bond than those of the C5 precursors, due to the greater length of their carbon chain. Further photolysis of such byproducts can eventually lead to increased generation of unsaturated $\mathrm{S}$ atoms 

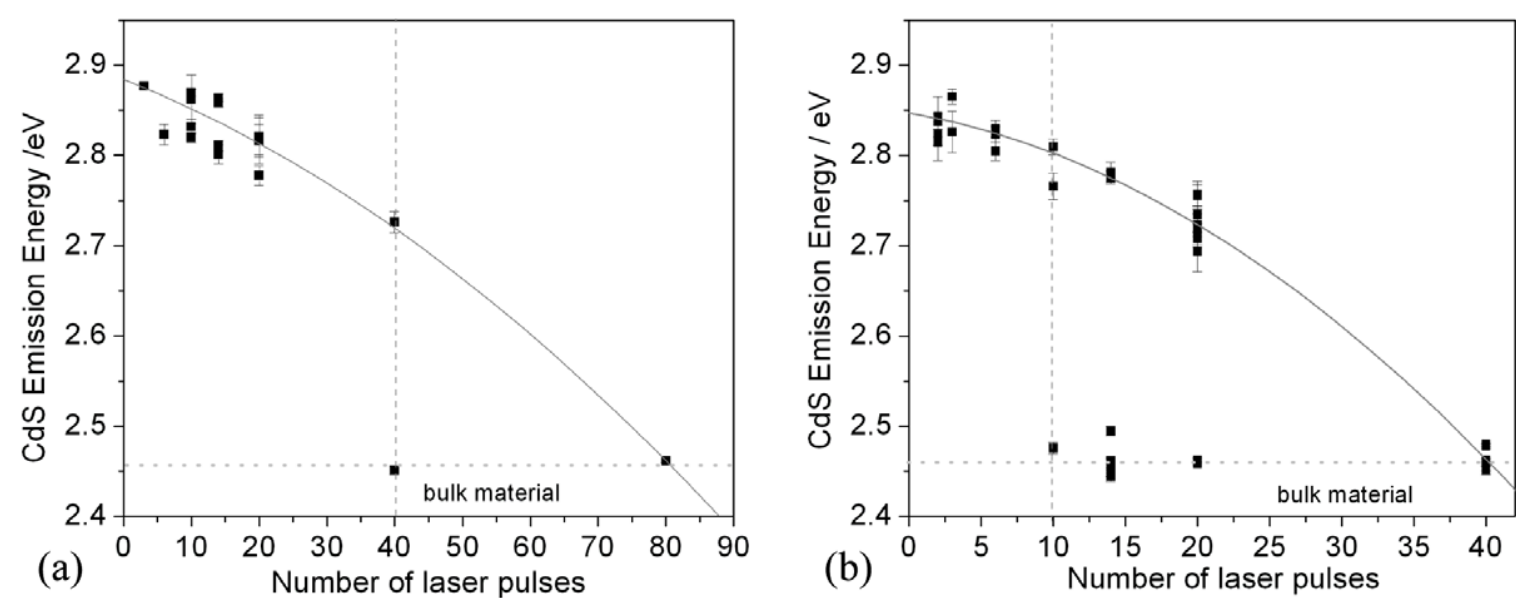

Figure 7. The evolution of the emission energy of the CdS NCs after irradiation of the C12-TOPAS (a) and C5-TOPAS films (b). The gray lines are guides for the eye.
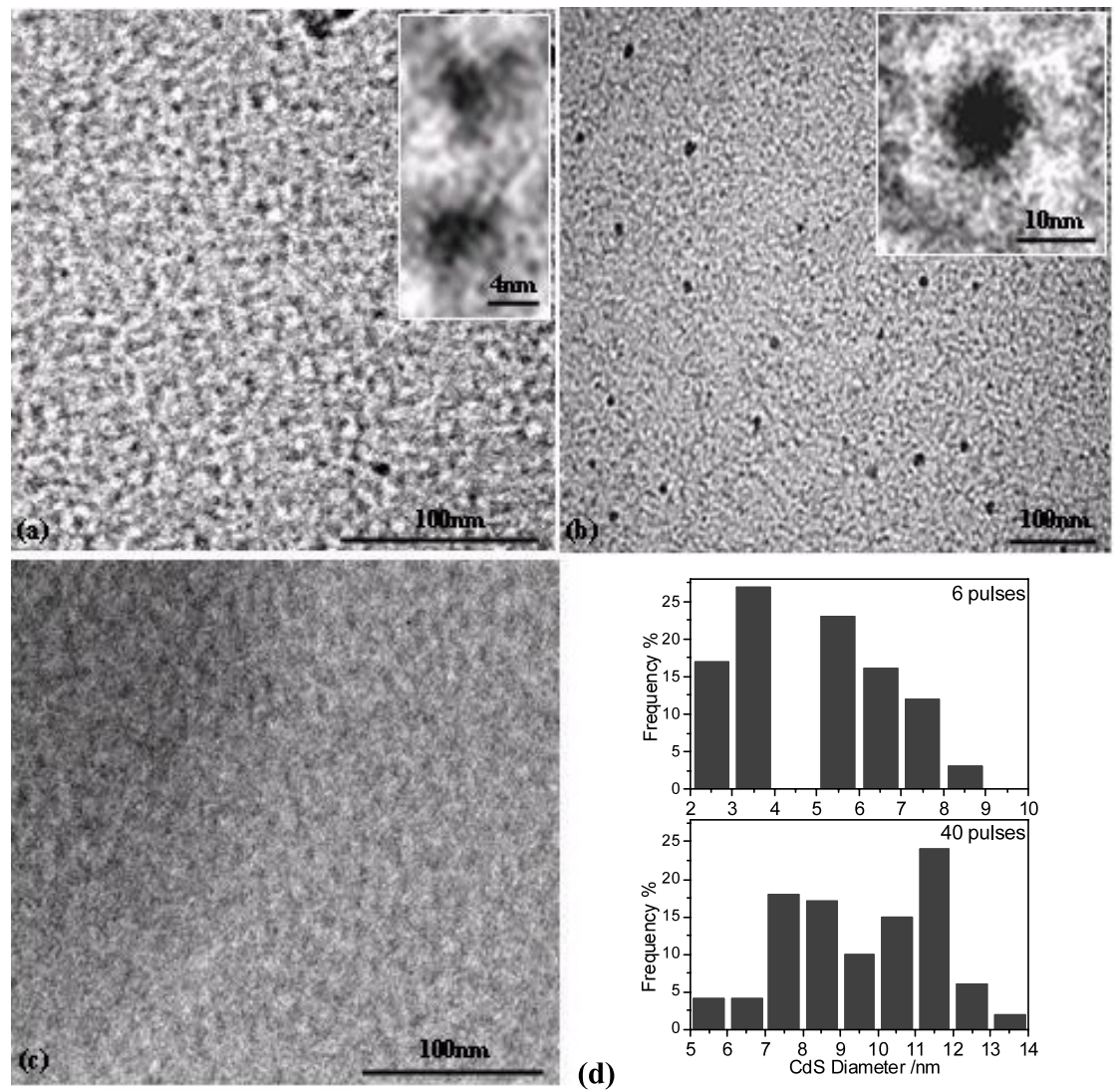

Figure 8. TEM images of CdS NCs formed after irradiation of C5-TOPAS films with UV light $(\lambda=266 \mathrm{~nm})$. After 6 pulses (a), after 40 pulses (b), and before any irradiation (c). (d) The histogram size distribution of the CdS NCs in each case.

in the $\mathrm{C} 12$ films, increasing the surface defects of the NCs, and consequently, their trap state emission. In the C12TOPAS films there is also the possibility of photoproduct formation of the polymer backbone chain that can lead to additional trap states. Since the absorption efficiency of the C12 precursor is lower than the C5 one, more photons can interact with the polymer chain in the former case under the same number of pulses, eventually leading to some polymer byproducts [34] that may induce surface defects to the NCs. Conclusively, the C5-TOPAS films give NCs with narrower emission spectra than those of the C12-TOPAS films, indicating a better quality of NCs with less surface defects.

\subsection{Patterned CdS nanocomposite formation}

Upon laser irradiation, it is expected that the NCs are localized exclusively in the irradiated area, making possible 
(a)

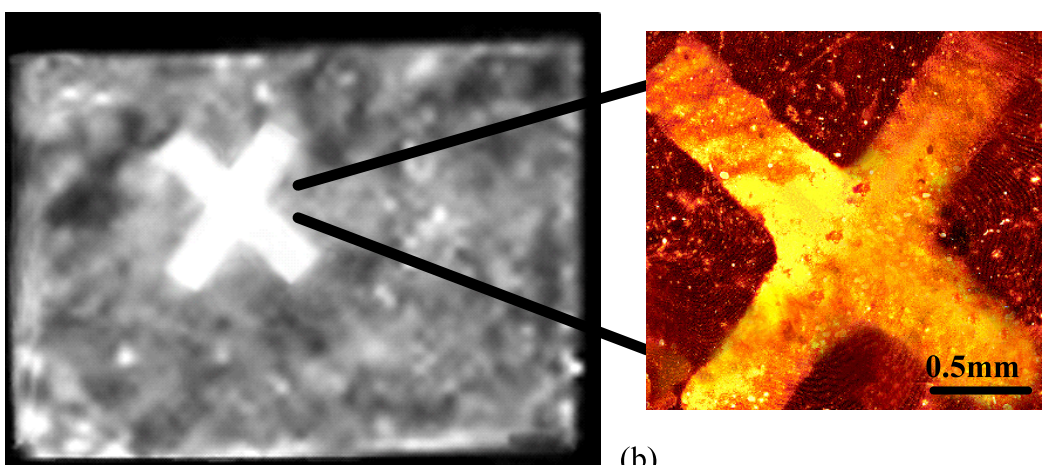

(b)

Figure 9. (a) Fluorescence image of a patterned sample of C12-TOPAS. The sample was excited with a UV lamp and the fluorescence was collected by using a long pass filter with a cut off wavelength at $480 \mathrm{~nm}$. (b) Fluorescence image of the same pattern after excitation with a $405 \mathrm{~nm}$ diode laser, with a magnification $5 \times$.

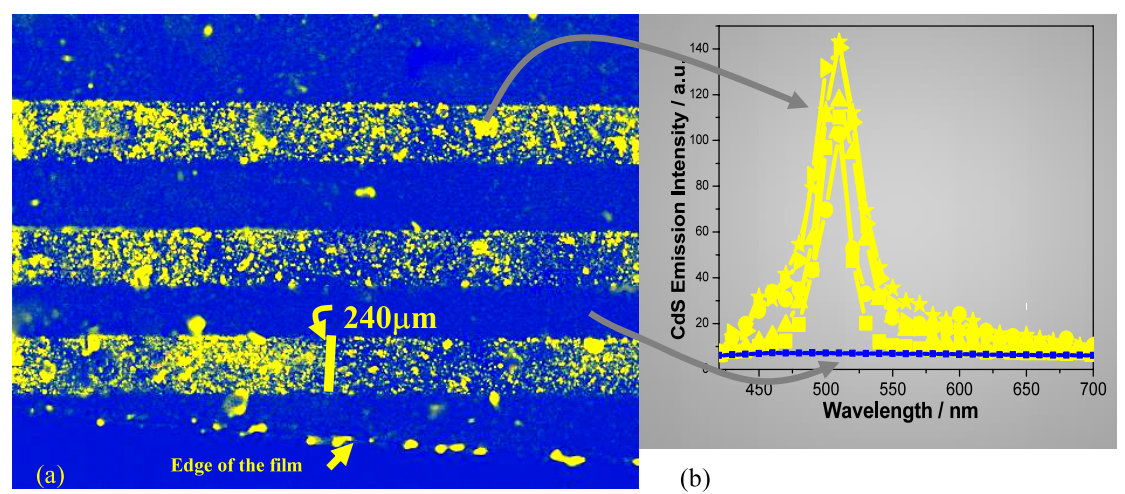

Figure 10. (a) Fluorescence image of a patterned sample of C12-TOPAS excited with a $405 \mathrm{~nm}$ diode laser and magnification $5 \times$. (b) The fluorescence spectra of each area.

the formation of patterns. Next, is reported the fabrication of patterns of CdS NC-structures in the TOPAS polymer matrix. Figure 9(a) illustrates the fluorescence image of a pattern of CdS NCs formed after irradiation of a C12-TOPAS film with 100 incident pulses through an ' $\mathrm{X}$ ' shaped mask with dimensions $5.0 \times 0.5 \mathrm{~mm}^{2}$. The pattern is apparent only under a UV lamp, since the polymer surface in the irradiated area remains macroscopically unaffected. In figure 9(b), the same pattern is observed under a fluorescence microscope with a magnification factor of $5 \times$ and after excitation with a pulsed diode laser at $405 \mathrm{~nm}$.

Figure 10(a) illustrates a micro pattern of 3 lines taken after focusing the laser beam on a C12-TOPAS sample fixed onto a motorized stage moving at constant velocity. The spot size of the beam is $\sim 0.2 \times 0.1 \mathrm{~mm}^{2}$, the repetition rate of the laser $10 \mathrm{~Hz}$, and the speed of the motor is about $0.2 \mathrm{~mm} \mathrm{~s}^{-1}$. The lines are fairly clear and their width $(240 \mu \mathrm{m})$ is constant along the irradiation path. The total number of pulses in each spot area is about 100 and is enough to form NCs with bulk properties, as demonstrated by their emission spectra shown in figure 10(b). The bright areas correspond to CdS NCs in the bulk region, since the spectrum has an emission peak at $506 \mathrm{~nm}$, while the dark areas, not being irradiated, have no fluorescence emission in the studied spectral region.

\section{Conclusions}

In summary, we have demonstrated the successful in situ formation of spatially and size controlled CdS NCs inside a polymer matrix, under pulsed UV laser irradiation. The optical properties of the formed nanocomposites depend on the number of incident pulses, while the selection of the precursor determines the efficiency of the procedure under the same irradiation conditions. In all the cases studied, the polymer remained macroscopically/microscopically unaffected, while the $\mathrm{CdS} \mathrm{NC}$ formation is already obtained at a small number of pulses. This method overrides the main disadvantage of the thermal annealing technique of similar systems, which is the non-localized formation of NCs inside the polymer matrix [24, 26, 35]. Furthermore, it is faster and much simpler than another method used for localized NC formation inside a polymer matrix, where hydrogen sulfide $\left(\mathrm{H}_{2} \mathrm{~S}\right)$ gas treatment is needed for about $48 \mathrm{~h}$ of a photopolymerized NC-precursor containing resin [36]. The technique presented opens up new ways for nanocomposite film patterning with size controlled NCs, and importantly, with controlled optical and physical properties. This fast and easy way is promising for the development of nanodevices for use in biotechnology or optoelectronics. 


\section{References}

[1] Duan X, Huang Y, Agarwal R and Lieber C M 2003 Nature 421241

[2] Grohs J, Apanasevich S, Jung P, Issler H, Burak D and Klingshirn C 1994 Phys. Rev. A 492199

[3] Shvydka D, Drayton J, Compaan A D and Karpov V G 2005 Appl. Phys. Lett. 87023505

[4] Wang Y 1994 J. Appl. Phys. 75332

[5] Schmitt-Rink S, Chemla D S and Miller D A B 1989 Adv. Phys. 3889

[6] Schlamp M C, Peng X G and Alivisatos A P 1997 J. Appl. Phys. 825837

[7] Chan Y, Steckel J S, Snee P T, Caruge J M, Hodgkiss J M, Nocera D G and Bawendi M G 2005 Appl. Phys. Lett. 86073102

[8] Achermann M, Petruska M A, Kos S, Smith D L, Koleske D D and Klimov V I 2004 Nature 429642

[9] Bruchez M Jr, Moronne M, Gin P, Weiss S and Alivisatos A P 1998 Science 2812013

[10] Chan W C W and Nie S M 1998 Science 2812016

[11] Empedocles S A, Norris D J and Bawendi M G 1996 Phys. Rev. Lett. 773873

[12] Li J and Wang L W 2003 Nano Lett. 31357

[13] Matsuura D, Kanemitsu Y, Kushida T, White C W, Budai J D and Meldrum A 2000 Appl. Phys. Lett. 772289

[14] Gerion D, Pinaud F, Williams S C, Parak W J, Zanchet D, Weiss S and Alivisatos A P 2001 J. Phys. Chem. B 105886

[15] Wang Y A, Li J J, Chen H and Peng X 2002 J. Am. Chem. Soc. 1242293

[16] Lee J, Sundar V C, Heine J R, Bawendi M G and Jensen K F 2000 Adv. Mater. 121102

[17] Chu Y-C, Wang C-C and Chen C-Y 2005 Nanotechnology 1658
[18] Tamborra M, Striccoli M, Comparelli R, Curri M L, Petrella A and Agostiano A 2004 Nanotechnology 15 S240

[19] Warner J H, Watt A A R and Tilley R D 2005 Nanotechnology 162381

[20] Mayer A B R 1998 Mater. Sci. Eng. C 6155

[21] Athanassiou A, Cingolani R, Tsiranidou E, Fotakis C, Laera A M, Piscopiello E and Tapfer L 2007 Appl. Phys. Lett. 91153108

[22] Fragouli D, Laera A M, Pompa P P, Caputo G, Resta V, Allione M, Tapfer L, Cingolani R and Athanassiou A 2008 Microelectron. Eng. at press doi:10.1016/j.mee.2008.12.050

[23] Henglein A 1989 Chem. Rev. 891861

[24] Di Luccio T, Laera A M, Tapfer L, Kempter S, Kraus R and Nickel B 2006 J. Phys. Chem. B 11012603

[25] Brus L E 1984 J. Chem. Phys. 804403

[26] Antolini F, Di Luccio T, Re M and Tapfer L 2005 Cryst. Res. Technol. 40948

[27] Scherrer P 1918 Göttin. Nachricht. 298

[28] Wang C-W and Moffit M G 2004 Langmuir 2011784

[29] Antoun T, Brayner R, Alterary S, Fiévet F, Chehimi M and Yassar A 2007 Eur. J. Inorg. Chem. 20071275

[30] Spanhel L, Haase M, Weller H and Henglein A 1987 J. Am. Chem. Soc. 1095649

[31] Brus L 1986 J. Phys. Chem. 902555

[32] Weller H 1993 Angew. Chem. Int. Edn 3241

[33] Carotenuto G, Martorana B, Perlo P and Nicolais L 2003 J. Mater. Chem. 132927

[34] Athanassiou A, Blasi L, De Giorgi M, Caputo G, Fragouli D, Tsiranidou E, Laera A M, Tapfer L and Cingolani R 2009 Polym. Compos. submitted

[35] Antolini F, Di Luccio T, Laera A M, Mirenghi L, Piscopielo E, Re M and Tapfer L 2007 Phys. Status Solidi b 2442768

[36] Sun Z-B, Dong X-Z, Chen W-Q, Shoji S, Duan X-M and Kawata S 2008 Nanotechnology 19035611 